\title{
Image-Guided Biopsies in the Head and Neck: Practical Value and Approach
}

$\mathbf{T}$ he authors of "Percutaneous CT-Guided Core Needle Biopsies of Head and Neck Masses: Technique, Histopathologic Yield, and Safety at a Single Academic Institution" have shown the efficacy and safety of imageguided core needle biopsy in the head and neck (H\&N) at their institution. A H\&N image-guided biopsy service adds immense value to patient care by offering an accurate and less invasive histopathologic diagnosis than a surgical biopsy, facilitating radiologic-pathologic correlation and growing a more management-driven radiology service. Three important points will underscore and highlight the practical value of the authors' work on image-guided core biopsy.

1. Head and neck fine-needle aspiration (FNA) versus core needle biopsy (CNB): When will FNA suffice and when is CNB likely to be needed?

2. The value of imaging biopsy versus surgical biopsy.

3. The value of the H\&N radiologist performing the biopsy.

In our head and neck biopsy service, we routinely do both ultrasound (US)-guided and CT-guided biopsies and both FNA and CNB. The referring clinician will request a biopsy; then, it comes to us for vetting. In general, US biopsies are more commonly requested and used for most superficial targets ( $<3 \mathrm{~cm}$ deep). CT-guided biopsies are usually reserved for deeper targets, most frequently near the skull base or in parapharyngeal or retropharyngeal spaces, which are not easily accessible with US.

The first choice in planning will be US versus CT:

\section{US}

- Targets $<3 \mathrm{~cm}$ deep

- Most lateral and supraclavicular lymph nodes

- Most parotid masses

- Thyroid and thyroid bed masses

\section{CT}

- Targets $>3 \mathrm{~cm}$ deep

- Lesions near the skull base/clivus
- Parapharyngeal space mass (often minor salivary tumors)

- Deep lobe of the parotid

- Retropharyngeal nodes

- Pterygopalatine fossa lesion

Some common indications for image-guided biopsy include a new palpable mass/node, staging nodes in a patient with known $\mathrm{H} \& \mathrm{~N}$ cancer including thyroid cancer, evaluating recurrence in nodes after treatment for $\mathrm{H} \& \mathrm{~N}$ cancer, and parotid or submandibular masses.

\section{HEN FNA versus Core Biopsy: When Will FNB Suffice and When Is CNB Needed?}

- FNA: Usually narrow-gauge 22 - to 25 -ga needle (23 ga most commonly)

1) A US guided FNA: $3.5 \mathrm{~cm} 23$ ga needle for most at our institution

2) Coaxial technique for CT-guided FNA, a 6-cm, 19-ga introducer and 10-cm, 22-ga needle for FNA

- CNB: Usually larger gauge needles (16-20 ga); SuperCore 18 ga for most at our institution (https://www.argonmedical.com/ products/supercore-semi-automatic-biopsy-instrument/).

The next important consideration is FNA versus CNB. The major benefit of FNA is safety, especially with small targets but also preliminary cytologic assessment by an on-site cytopathologist, allowing real-time feedback regarding the adequacy of the sample. The main disadvantage of FNA is the small sample size, which can lead to nondiagnostic results. FNAs are generally performed with narrow-gauge needles (22-25 ga), and core needle biopsies are performed with larger gauge needles (16-20 ga). The main advantage of CNB is the increased diagnostic yield compared with FNA of salivary gland lesions and cervical lymph nodes. ${ }^{1,2}$

There will be some circumstances when the referring team (or the radiologist) will request a core upfront. Some reasons would include a previous non-diagnostic FNA, a suspected diagnosis that is difficult to make on FNA alone or enrollment in a clinical trial which needs core tissue. In addition, if asked to biopsy a lesion with a less typical leading diagnosis, it is often helpful to 
discuss the case ahead of time with your cytopathologists to understand their comfort level making that particular diagnosis with FNA vs CNB. At our institution, most biopsies will start with FNA and then progress to core on the basis of the real-time feedback of the cytopathologist. At some institutions, a CNB may also be chosen over FNA because of the preference of the radiologists or availability and expertise of the cytopathologists. However, it is useful for radiologists to have a general concept of which diagnoses can be easily made with FNA and which will likely require core. This is important to understand before starting a biopsy.

In our experience, FNA alone will usually suffice to diagnose squamous cell carcinoma (SCC), papillary thyroid carcinoma (with thyroglobulin assay), melanoma, and other carcinomas, particularly in patients who already have a confirmed head and neck primary. Other histopathologic diagnoses are more difficult to make with FNA. For example, salivary gland neoplasms and infiltrative soft-tissue masses around the skull base (with a differential of lymphoma, lymphoma mimics, immunoglobulin G4, sarcoidosis, chronic infection, etc.) and are all examples of lesions which can be difficult to diagnose on FNA.

In general, FNA has a high diagnostic yield for SCC often in the setting of tumor recurrence, thyroid carcinoma, and melanoma. It is often our choice for small lesions in this category. In fact, a retrospective review of our preliminary institutional experience with a $H \& N$ biopsy service in 2010 found that 88/102 $(87 \%)$ FNAs (mean target size $=1.6 \mathrm{~cm}$ ) performed in the nonthyroid neck were diagnostic. ${ }^{3}$ The clinical indications for USguided FNA included an initial diagnosis of a nonthyroid gland neck mass $(n=13)$, initial cervical lymph node staging in the setting of known malignancy $(n=3)$, suspected abscess $(n=4)$, and suspected persistence or recurrence of a known malignancy $(n=75)$. The latter category was clearly the most common indication, with the most common histopathology being thyroid carcinoma, squamous cell carcinoma, and melanoma in descending order.

FNA has a higher non-diagnostic rate and limited sensitivity for malignancy in salivary gland neoplasms. FNA can also be more challenging with lymphoma and rare inflammatory diagnoses as noted above. CNB of salivary gland lesions has emerged as the preferred method to overcome the limitations of FNA. In 3 studies directly comparing FNA and CNB of salivary lesions, the nondiagnostic rate was $19 \%-56 \%$ for FNA and $4 \%-5 \%$ for $\mathrm{CNB}$, while the sensitivity for malignancy was $60 \%-76 \%$ for FNA and 89\%-93\% for CNB, with only minor complications such as hematoma and no major complication such as facial nerve injury. ${ }^{1-5}$ Compared with FNA alone, CNB has also been shown to increase the diagnostic yield for lymphoma and decrease the need for excisional biopsy. ${ }^{6}$

At our institution, we often begin with FNA for both salivary neoplasms and suspected lymphoma, but we have a lower threshold to add CNB if there is a sufficient-size solid component and preliminary FNA is nondiagnostic. We use a SuperCore 18-ga core biopsy gun with an adjustable specimen notch for either 1- or 2-cm core specimens. The needle has an echogenic tip on the outer cannula to promote accurate placement under ultrasound guidance.
What is the Value of Image-Guided Biopsy versus Surgical Biopsy?

Many patients with head and neck cancer have other comorbidities, which make open biopsy under general anesthesia a higher risk. In addition, patients who have already undergone complex resection, reconstruction, and/or radiation also have tissue distortion and scarring, which complicates a re-operation. Finally, most of these deep abnormalities are detected by imaging only. Therefore, imageguided biopsy ensures radiologic-pathologic correlation. In other words, image-guided biopsy ensures that the suspicious lesion on imaging is actually the tissue that is correctly sampled. In a deep resection bed with extensive scarring/granulation and flap reconstruction, it is not a simple task for the surgeon to confirm that he or she is sampling the imaging abnormality, and this issue can lead to false-negatives.

\section{What is the Value of a Diagnostic HEN Radiology Group Performing the Biopsies for the Studies They Interpret?}

Divorcing the interpreting radiologists from the radiologist performing the biopsies can affect both the accuracy of the original image interpretations and the accuracy of the biopsies (confusion over target/exact area of concern in some cases). Diagnostic radiologists who do not perform procedures and/or communicate with colleagues performing the procedures may overcall imaging abnormalities which are difficult to access. An imaging abnormality with an unclear risk assessment that cannot easily be biopsied can lead to frustration for the referring clinician and stress for the patient. Radiologists who routinely perform their own biopsies quickly develop an understanding of whether they can safely reach the target, whether an abnormality can be successfully biopsied, whether a CT abnormality will translate to a viable US target, whether the target is reproducible, and so forth. It can be very beneficial to think like an interpreting radiologist and a procedure-vetting radiologist at the time of your original dictation.

There are many potential advantages to having a neuroradiologist or dedicated head and neck radiologist perform the imageguided biopsy. Thorough knowledge of the neck imaging anatomy, normal and abnormal appearance of the postoperative/postradiotherapy neck, and specific patterns of nodal spread for various head and neck malignancies are major advantages. The neuroradiologist often interprets the imaging or participates in multidisciplinary tumor boards that prompt FNA, leading to familiarity with the case and deliberation on the advantages and disadvantages of US-guided FNA, CT-guided FNA, and core or follow-up imaging. This scenario is particularly important in complex cases with $>1$ potential target. The true risk category of an imaging abnormality may be at the crossroads of multiple modalities (CT, PET, MR imaging) and multiple time points. Thus, the accuracy of biopsies can also be adversely affected if the interpreting radiologist is not performing the biopsy. There is also an opportunity to reconcile radiologic-pathologic discordance on site during the procedure, so that mismatches can be discussed and additional passes/alternative targets selected during the procedure. This interaction also facilitates a collegial relationship among the head and neck surgeon, neuroradiologist, and cytopathologist, which is the key to optimal multidisciplinary patient care. 
In summary, image-guided biopsies have replaced surgical biopsies for most head and neck lesions, with increased safety and efficacy. Furthermore, the many potential advantages of having a neuroradiologist or dedicated head and neck radiologist perform the image-guided biopsy include thorough knowledge of the neck anatomy, normal and abnormal appearance of the postoperative/ postradiotherapy neck, and specific patterns of nodal spread for various head and neck malignancies. While FNA will usually suffice to establish cervical nodal metastasis or nodal recurrence for common pathologies such as SCC, thyroid carcinoma, and melanoma, CNB may be needed for increased accuracy for salivary gland masses and suspected lymphoma. In the end, the decision to use FNA versus CNB is often based on the unique presentation of each case, the operator's experience, institutional preference, and real-time cytopathologic evaluation.

\section{REFERENCES}

1. Eom HJ, Lee JH, Ko MS, et al. Comparison of fine-needle aspiration and core needle biopsy under ultrasonographic guidance for detecting malignancy and for the tissue-specific diagnosis of salivary gland tumors. AJNR Am J Neuroradiol 2015;36:1188-93 CrossRef Medline
2. Novoa E, Gurtler N, Arnoux A, et al. Role of ultrasound-guided core-needle biopsy in the assessment of head and neck lesions: a meta-analysis and systematic review of the literature. Head Neck 2012;34:1497-1503 CrossRef Medline

3. Lorenzo G, Saindane AM, Aiken A, Lewis M, Hollis B, Hudgins PA. Diagnostic utility of ultrasound-guided fine needle aspiration in the (non-thyroid gland) neck. American Society of Head and Neck Radiology, Houston, TX, October 2010 (oral presentation).

4. Pratap R, Qayyum A, Ahmed N, et al. Ultrasound-guided core needle biopsy of parotid gland swellings. J Laryngol Otol 2009;123:449-52 CrossRef Medline

5. Haldar S, Mandalia U, Skelton E, et al. Diagnostic investigation of parotid neoplasms: a 16-year experience of freehand fine needle aspiration cytology and ultrasound-guided core needle biopsy. Int J Oral Maxillofac Surg 2015;44:151-57 CrossRef Medline

6. Ryu YJ, Cha W, Jeong WJ, et al. Diagnostic role of core needle biopsy in cervical lymphadenopathy. Head Neck 2015;37:229-33 CrossRef Medline

(1) A.H. Aiken

Departments of Radiology and Imaging Sciences and Otolaryngology-Head and Neck Surgery Emory University Atlanta, Georgia 